TITLE:

\title{
Ab Initio Analysis of Auger-Assisted Electron Transfer
}

$\operatorname{AUTHOR}(S)$ :

Hyeon-Deuk, Kim; Kim, Joonghan; Prezhdo, Oleg V.

CITATION:

Hyeon-Deuk, Kim ...[et al]. Ab Initio Analysis of Auger-Assisted Electron Transfer. Journal of Physical Chemistry Letters 2015, 6(2): 244-249

ISSUE DATE:

2015-01-15

URL:

http://hdl.handle.net/2433/253739

RIGHT:

This document is the Accepted Manuscript version of a Published Work that appeared in final form in Journal of Physical Chemistry Letters, copyright (C) American Chemical Society after peer review and technical editing by the publisher. To access the final edited and published work see https://doi.org/10.1021/jz502505m.; This is not the published version. Please cite only the published version.; この論文は出版社版でありません。引用の際には出版社版 をご確認ご利用ください。 


\title{
Ab initio Analysis of Auger-Assisted Electron
}

\section{Transfer}

\author{
Kim Hyeon-Deuk, ${ }^{*},+\ddagger$ Joonghan Kim, and Oleg V. Prezhdo*,§ \\ Department of Chemistry, Kyoto University, Kyoto, 606-8502, Japan, Japan Science and \\ Technology Agency, PRESTO, 4-1-8 Honcho, Kawaguchi, Saitama, 332-0012, Japan, \\ Department of Chemistry, The Catholic University of Korea, Bucheon, Gyunggi-do \\ 420-743, Korea, and Department of Chemistry, University of Southern California, Los \\ Angeles, CA 90089, USA
}

E-mail: kim@kuchem.kyoto-u.ac.jp; prezhdo@usc.edu

\footnotetext{
*To whom correspondence should be addressed

${ }^{\dagger}$ Department of Chemistry, Kyoto University, Kyoto, 606-8502, Japan

${ }_{\ddagger}^{\ddagger}$ Japan Science and Technology Agency, PRESTO, 4-1-8 Honcho, Kawaguchi, Saitama, 332-0012, Japan

IDepartment of Chemistry, The Catholic University of Korea, Bucheon, Gyunggi-do 420-743, Korea

$\S$ Department of Chemistry, University of Southern California, Los Angeles, CA 90089, USA
} 


\begin{abstract}
Quantum confinement in nanoscale materials allows Auger-type electron-hole energy exchange. We show by direct time-domain atomistic simulation and analytic theory, that Auger processes give rise to a new mechanism of charge transfer (CT) on the nanoscale. Auger-assisted CT eliminates the renown Marcus inverted regime, rationalizing recent experiments on CT from quantum dots to molecular adsorbates. The ab initio simulation reveals a complex interplay of the electron-hole and charge-phonon channels of energy exchange, demonstrating a variety of CT scenarios. The developed Marcus rate theory for Auger-assisted CT describes, without adjustable parameters, the experimental plateau of the CT rate in the region of large donor-acceptor energy gap. The analytic theory and atomistic insights apply broadly to charge and energy transfer in nanoscale systems.
\end{abstract}

\title{
TOC Graphic.
}

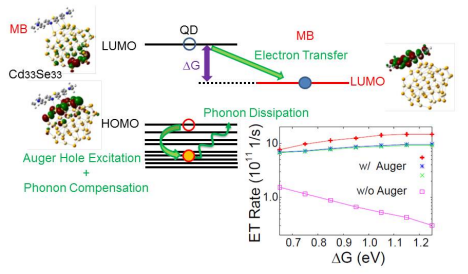

Keywords: Auger-Assisted Electron Transfer, Carrier Separation, Quantum Dots, ElectronPhonon Couplings, Fermi's golden rule 
Quantum confinement of charge carriers in semiconductor nanoscale materials, such as quantum dots (QDs) and nanotubes, leads to novel phenomena, giving rise to new classes of systems utilized for light harvesting and charge separation during solar energy conversion. ${ }^{1-4}$ The rates and mechanisms of photo-generation and dissociation of coupled electron-hole pairs, known as excitons, at interfaces of nanoscale materials with various charge acceptors determine efficiencies of photovoltaic and photo-catalytic devices. ${ }^{5-8}$ One customarily invokes Marcus theory ${ }^{9-12}$ to describe electron transfer(ET) in order to get the ET rate as a function of the electron donor-acceptor(D-A) energy gap, known as the driving force, and the D-A couplings. Developed initially for intra- and inter-molecular ET, Marcus theory assumes that ET is accompanied by a large rearrangement of nuclear configuration, which brings the electronic donor and acceptor energies in resonance. The energy of the nuclear rearrangement is called the reorganization energy. The ET rate in traditional Marcus theory reaches a maximum when the reorganization energy matches the D-A energy gap. If the gap becomes too large, the nuclear rearrangement requires significant thermal activation, and the rate decreases, leading to the celebrated Marcus inverted region. ${ }^{10}$ The recent experimental studies on the complexes of QD donors with molecular acceptors reported ET rates over a broad range of driving energies. ${ }^{13}$ The rates of the photo-induced ET from the QDs to the molecular acceptors grew with increasing driving force, finally reaching a plateau. The expected Marcus inverted region was not detected, suggesting possibility for ultra-efficient charge transfers in nanoscale confined materials. ${ }^{14}$

In this letter, we report time-domain ab initio simulation of ET from a CdSe QD to an adsorbed methylene blue(MB) molecule, faithfully representing the experimental system. ${ }^{14}$ The simulation has demonstrated that the ET process is accompanied by Auger-type electronhole energy exchange, rationalizing the lack of the inverted regime in the experimental data. The current simulation reveals a complex interplay of the electron-hole and charge-phonon channels of energy exchange, demonstrating a variety of electron transfer (ET) scenarios. It shows that the traditional ET mechanism mediated by phonons competes with the Auger- 
assisted ET process. A notable fraction of the electron energy flows directly to phonons, bypassing the hole. Phonons dissipate the excited hole energy, allowing the hole to accommodate additional electron energy. Having lost some energy to phonons, the hole can be excited again, facilitating the ET further. In addition to the atomistic simulation which exhibits a complex interplay between the electron, hole and phonon dynamics following QD photo-excitation, we derive an analytic theory of Auger-assisted electron transfer (AAET) in this letter. Based solely on ab initio input and without any adjustable parameters, the developed theory successfully reproduces the experimentally observed plateaus in the ET rate at large driving forces.

The electronic structure and adiabatic MD are computed with VASP with the PW91 density functional and projector-augmented-wave pseudopotentials as explained in the Supporting Information. The $\mathrm{QD} / \mathrm{MB}$ complex is fully optimized at zero temperature and heated up to an ambient temperature by repeated velocity rescaling. A 4 ps microcanonical trajectory is calculated on the ground electronic state by the ab initio MD using the Verlet algorithm. It is hard to know how MB molecules are attached to QDs in the experiments. It is likely that the MB molecules are directly bound to QDs, while other areas of the QD surface are covered by ligands. Our ab initio MD simulations show that the MB molecule is adsorbed and remains stable on the QD surface in spite of large thermal and structural fluctuations at the ambient temperature.

In order to account for the Auger hole excitation accompanying the ET, we adopt the exciton representation. The driving force of the ET, $\Delta G$, is defined as the energy difference between the QD LUMO and the MB LUMO. The non-adiabatic(NA) dynamics simulations for the AAET are performed by combining the time-domain DFT(TDDFT) with the NAMD. ${ }^{15}$ The adiabatic KS orbitals are obtained with the DFT for atomic positions at each moment along the ab initio MD trajectory. We represent the original TDDFT equations in the second quantization notation to include the ground and single exciton (SE) states, 
$\left|\Phi_{g}(\mathbf{r} ; \mathbf{R})\right\rangle$ and $\left|\Phi_{S E}^{i, j}(\mathbf{r} ; \mathbf{R})\right\rangle$, respectively. The total wave function is then expanded as

$$
|\Psi(t)\rangle=C_{g}(t)\left|\Phi_{g}\right\rangle+\sum_{i, j} C_{S E}^{i, j}(t)\left|\Phi_{S E}^{i, j}\right\rangle
$$

Substitution of eq.(1) into the time-dependent Schrödinger equation leads to the EOMs for the expansion coefficients,

$$
i \hbar \frac{\partial C_{X}(t)}{\partial t}=C_{X}(t) E_{X}-i \hbar C_{g}(t) \mathbf{d}_{X ; g} \cdot \dot{\mathbf{R}}-i \hbar \sum_{i^{\prime}, j^{\prime}} C_{S E}^{i^{\prime}, j^{\prime}}(t) \mathbf{d}_{X ; S E, i^{\prime}, j^{\prime}} \cdot \dot{\mathbf{R}}
$$

where $X$ corresponds to either the ground or SE state, and $E_{X}$ is the state energy. The NA couplings introduced by

$$
\mathbf{d}_{X ; Y} \cdot \dot{\mathbf{R}} \equiv\left\langle\Phi_{X}\left|\nabla_{\mathbf{R}}\right| \Phi_{Y}\right\rangle \cdot \dot{\mathbf{R}}=\left\langle\Phi_{X}\left|\frac{\partial}{\partial t}\right| \Phi_{Y}\right\rangle
$$

induce NA transitions between the states in the exciton basis. The atomistic simulation of the ET dynamics is performed by directly solving eq.(2) with the time-dependent NA couplings (3) and energies obtained by the ab initio MD simulation. We further introduced energy dissipation to phonon modes by the exponential decay function, $\exp \left(-t / \tau_{\text {diss }}\right)$, where $t$ is a simulation time and $\tau_{\text {diss }}$ is a typical dissipation timescale. ${ }^{16}$ 


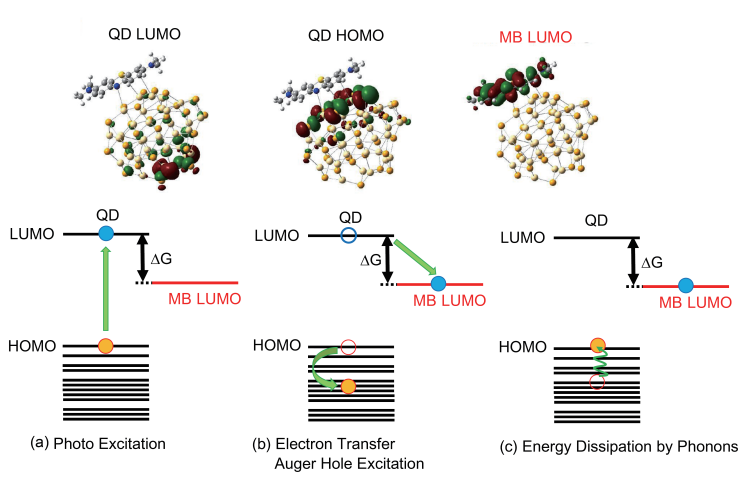

Figure 1: (Upper) Densities of the key orbitals participating in the AAET. (Lower) Mechanism of the AAET. The electron and hole is initially excited in the QD CB and VB, respectively. The hole excitation in the QD VB accompanies the ET from the QD to the MB. This mechanism becomes possible in a QD due to strong Coulomb interaction between the confined electron and hole, and a high density of hole states. The excess energy which the hole gained is finally dissipated by phonons.

A schematic of the ET involving the Auger hole excitation is given in Fig. 1. Photoexcitation promotes an electron from the QD HOMO to the QD LUMO, leaving a hole in the QD HOMO. Since the QD+MB complex is asymmetric, it is natural that the orbitals are distributed asymmetrically. In the current case, the CdSe QD attracts the electron more than the MB molecule, making the QD HOMO localized close to the MB and the QD LUMO localized far from the MB.

We focus on the ET from the $\mathrm{Cd}_{33} \mathrm{Se}_{33} \mathrm{QD}$ to the adsorbed MB molecule. The ET involves an electronic transition from the QD LUMO to the MB LUMO; the electron traverses the entire QD. In the traditional ET, nuclear vibrational motions accommodate the excess energy lost by the electron. In the current system, the energy lost by the electron is taken up by the hole which is promoted from the QD HOMO into the deeper valence band (VB) of the QD. The quantum confinement of the charge carriers in the QD enhances their Coulomb interaction, allowing this electron-hole energy exchange, and opening up an alternative pathway for the energy flow. In addition, since the QD HOMO is located close to the MB, it is strongly coupled to the high-frequency MB phonon modes, inducing the efficient hole excitation. The excess energy transferred from the electron to the hole is ulti- 
mately dissipated by phonon modes of the CdSe QD/MB complex; excited states of the hole, located energetically deep inside the $\mathrm{VB}$, tend to delocalize over the whole QD, in contrast to the QD HOMO. The Auger mechanism is inefficient in bulk semiconductors because of weak, screened Coulomb interactions. There has been no observation of the AAET in any bulk semiconductor. The Marcus inverted region appears in such cases. Electron-hole interactions are affected by material-dielectric properties. Electrons and holes weakly interact in semiconductors, forming excitons. The exciton Bohr radius determines the material size at which the quantum confinement effects appear. The Coulomb interactions can be strong in molecules; however, the densities of electron and hole states are low. Low state densities reduce Auger processes, making it hard to match properly the electron and hole energy levels. 


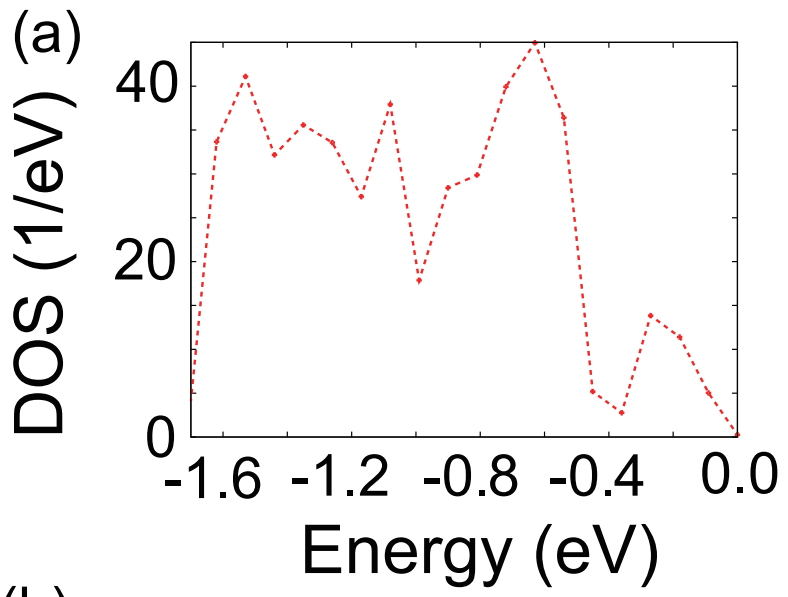

(b)

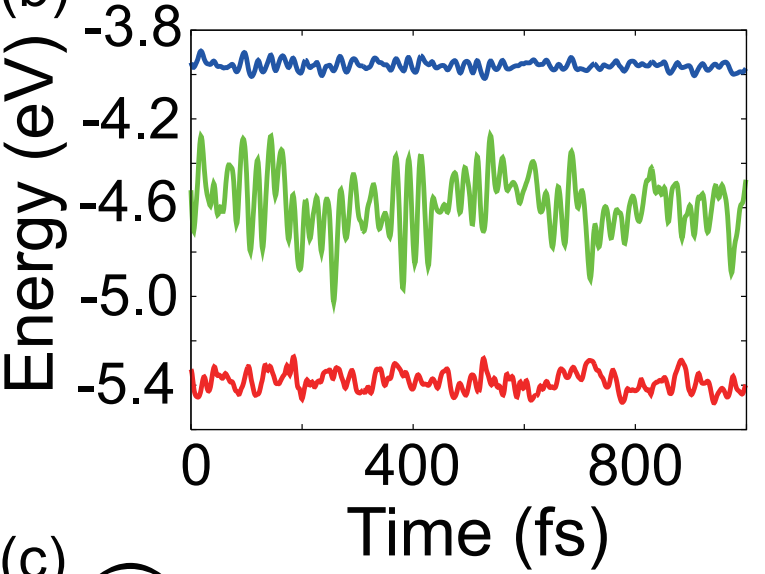

(c)

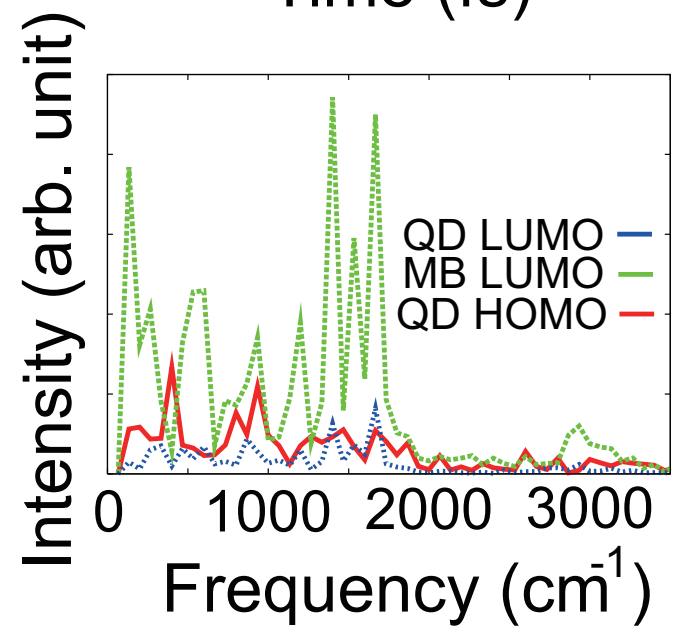

Figure 2: (a) VB DOS of the $\mathrm{Cd}_{33} \mathrm{Se}_{33}$ QD. The VB DOS increases away from the band gap and then saturates. (b) Energy fluctuations of the QD HOMO(red line), LUMO(blue line) and MB LUMO(green line). The fluctuation amplitude reflects the strength of the electronphonon coupling. (c) The Fourier transforms of the evolving orbital energies characterize the phonon modes that couple to the electronic subsystem. The MB LUMO exhibits the strongest phonon intensity as expected, while the phonon intensity of the QD LUMO is weakest. 
The VB density of states (DOS) is particularly important for the AAET. We averaged $a b$ initio molecular dynamics (MD) data of 2 ps on the VB energies fluctuating at the ambient temperature, and obtained the VB DOS shown in Fig. 2(a). It exhibits non-trivial dependence, with growing and then saturating away from the Fermi energy, $E_{F}=0$. The dependence is neither square root, following from the simple parabolic dispersion model, nor exponential, expected for a distribution of surface defect states. The saturation is essential to reproduce the experimental plateau of the AAET rates, ${ }^{14}$ as will be shown later. Figure 2(b) demonstrates that the energy of the QD HOMO fluctuates more than the energy of the QD LUMO. This is because the QD HOMO is spatially close to the MB molecule and is affected by the molecular vibrations. The MB LUMO energy fluctuates most because the molecule undergoes larger scale motions than the QD. The Fourier transforms of the evolving orbital energies characterizing the phonon modes that couple to the electronic subsystem is given in Fig. 2(c). The phonon spectrum for the acceptor state, the MB LUMO, exhibits strong intensity for the high frequency modes, indicating that these modes actively participate in the ET by the traditional mechanism, which competes with the Auger process. The large phonon intensity of the QD HOMO also suggests that the high frequency molecular modes effectively assist relaxation and excitation of the hole. 

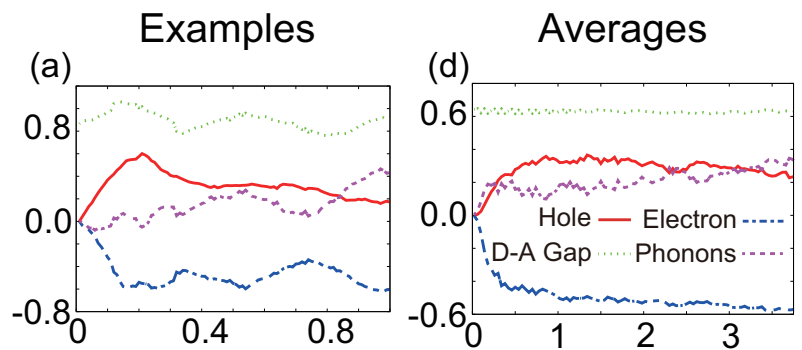

(b)

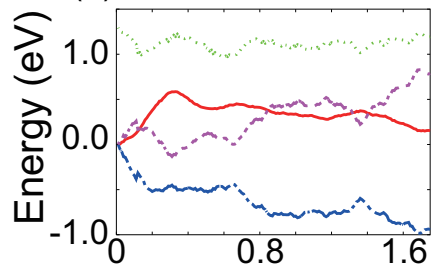

(e)

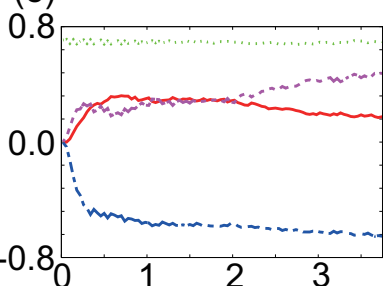

(c)

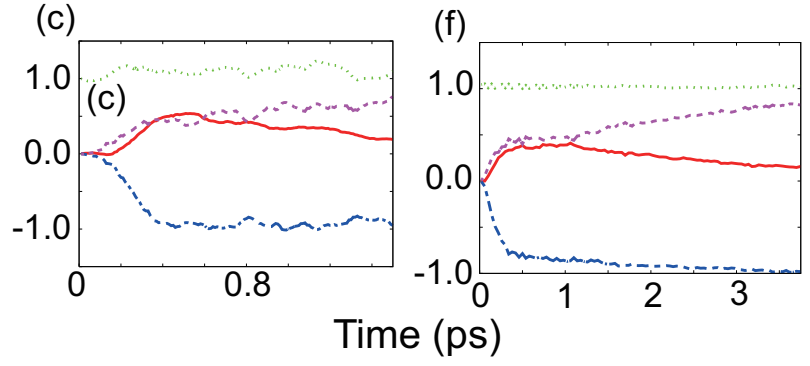

Figure 3: (a)-(c) Representative dynamics of the energies stored by the electron(blue line), hole(red line), phonons(purple line), and the electron D-A energy gap(green line) during the AAET whose schematic illustration is given in Fig. 1. The energy lost by the electron is accommodated by both the hole and phonons. At a longer time, the hole relaxes by donating its energy to phonons. (d)-(f) Dynamics averaged over 51 non-equilibrium MD trajectories for different D-A energy gaps. The excess energy generated by the ET is only partially accommodated by the hole, and the rest of the electron energy is compensated by phonons. At a later stage, the ET continues at a slower pace, and the hole starts to lose energy to phonons by dissipation. The shown data include both statistical mechanical and quantum mechanical averaging.

Figure 3(a)-(c) shows time evolutions of the electron, hole and phonon energies along representative non-equilibrium AAET trajectories initiated by electron excitation from the QD HOMO to the QD LUMO. The D-A energy gap, i.e. the energy difference between the QD LUMO and MB LUMO, is shown as well. As the ET proceeds, the electron energy decreases on a picosecond timescale, in agreement with the experimental data. ${ }^{14}$ In the initial stage of Fig. 3(a), the energy lost by the electron is gained exclusively by the hole, confirming the AAET mechanism. At a later time, after the electron has been transferred and the hole 
has been excited, the hole deposits its excess energy to phonons. This Auger-type electronhole energy exchange assisting the ET is not always perfect, however. At the earliest stage in Fig. 3(b), the phonons gain more energy than the hole. By the time when the ET signal has reached a plateau, around $0.2 \mathrm{ps}$, the hole has captured all of electron's energy, while, on the longer timescale, the hole loses energy to phonons. Figure 3(c) shows an example in which the hole and phonons participate equally in the energy exchange during the ET event. This example demonstrates that phonons take active part in the AAET process. In neither case, the hole gains the energy equal to the D-A energy gap because of dissipation by phonons. In the long-time limit, the electron energy decreases by the D-A energy gap, the hole energy converges to zero, and the phonon energy approaches to the D-A energy gap.

The above insights obtained in the single trajectory cases are also confirmed by the averaged data. Panels (d)-(f) of Fig. 3 display the dynamics averaged over 51 non-equilibrium MD trajectories for different D-A energy gaps. The excess energy generated by the ET is only partially accommodated by the hole, and the rest of the electron energy is compensated by phonons. At a later stage, the ET continues at a slower pace, and the hole starts to lose energy to phonons by dissipation. The data shown in Fig. 3(d)-(f) include both statistical mechanical and quantum mechanical averaging, while the data of Fig. 3(a)-(c) are averaged only quantum mechanically. The results averaged quantum mechanically can be interpreted statistically as well. The time-dependent data shown in the examples, Fig. 3(a)-(c), can be viewed as probabilities for different opportunities of the system to have the given energies. For instance, the energy of the electron Fig. 3(a) decreases rapidly and nearly linearly within the first $0.15 \mathrm{ps}$. The result can be interpreted that there is a $1 / 3$ chance that the electron hops within the first $0.05 \mathrm{ps}$, a $1 / 3$ chance that it hops between 0.05 and $0.1 \mathrm{ps}$, and a $1 / 3$ chance that it hops between 0.1 and $0.15 p s$. The hole energy increases rapidly and linearly on the same timescale, and the hole evolution can be interpreted in the same way. 


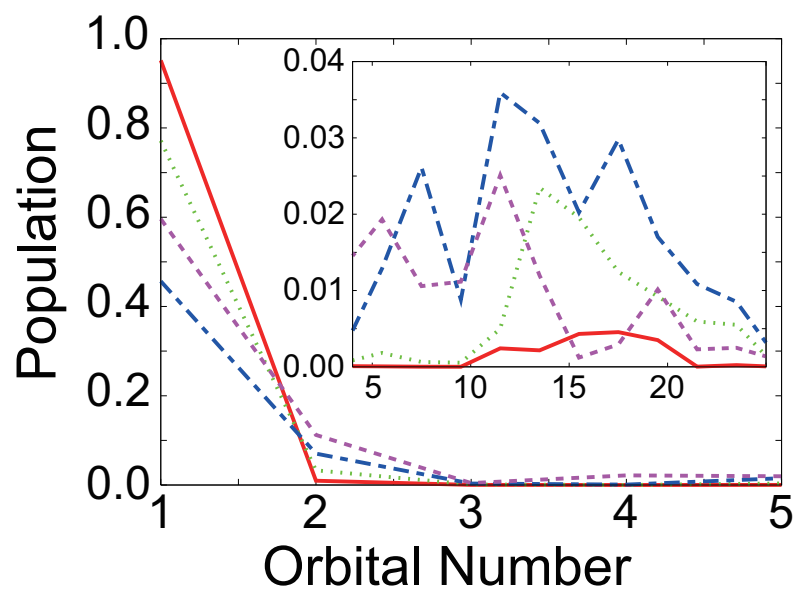

Figure 4: Distribution of the hole among different VB orbitals at 0.1 ps(red line), 0.2 ps(green line), $0.5 \mathrm{ps}$ (blue line), and $3.8 \mathrm{ps}$ (purple line) following the photo-excitation. The highest five VB orbitals starting with the QD HOMO (orbital number 1) are shown in the main panel, while the insert shows occupations of deeper orbitals. The orbital number in the $\mathrm{x}$-axis correlates with the orbital energy below the QD VB edge. The Auger hole excitation during the ET is directly demonstrated by the population shift to the deeper VB states. At $3.8 \mathrm{ps}$, the hole is already relaxing back to the QD HOMO.

Promotion of the hole from the QD HOMO into the deeper QD VB during the ET is directly demonstrated in Fig. 4. The distributions of hole populations in different VB orbitals are shown for various elapsed times. The x-axis denotes the VB orbital number, with 1 corresponding to the QD HOMO. At time $t=0$, the hole is in the QD HOMO. At $0.1 \mathrm{ps}$, the hole still populates the QD HOMO. The initial slow stage of quantum population transfer is generic to all quantum transitions. Demonstrated more explicitly in Fig.S2 of the Supporting Information, this Gaussian, or parabolic, or cosine regime can be viewed as part of a Rabi oscillation, giving rise to the quantum Zeno effect. ${ }^{14,17-25}$ At later times, the hole distribution shifts to orbitals deeper inside the VB. The population shift directly demonstrates the Auger hole excitation during the ET; the excess energy generated by the ET is actually accommodated by the hole. The hole excitation is not uniform because the energy levels of the VB states are discrete, and the NA couplings connecting each state pair vary in both energy and time. At long times, the hole distribution flows back to the upper VB states, reflecting hole energy dissipation by phonons. 

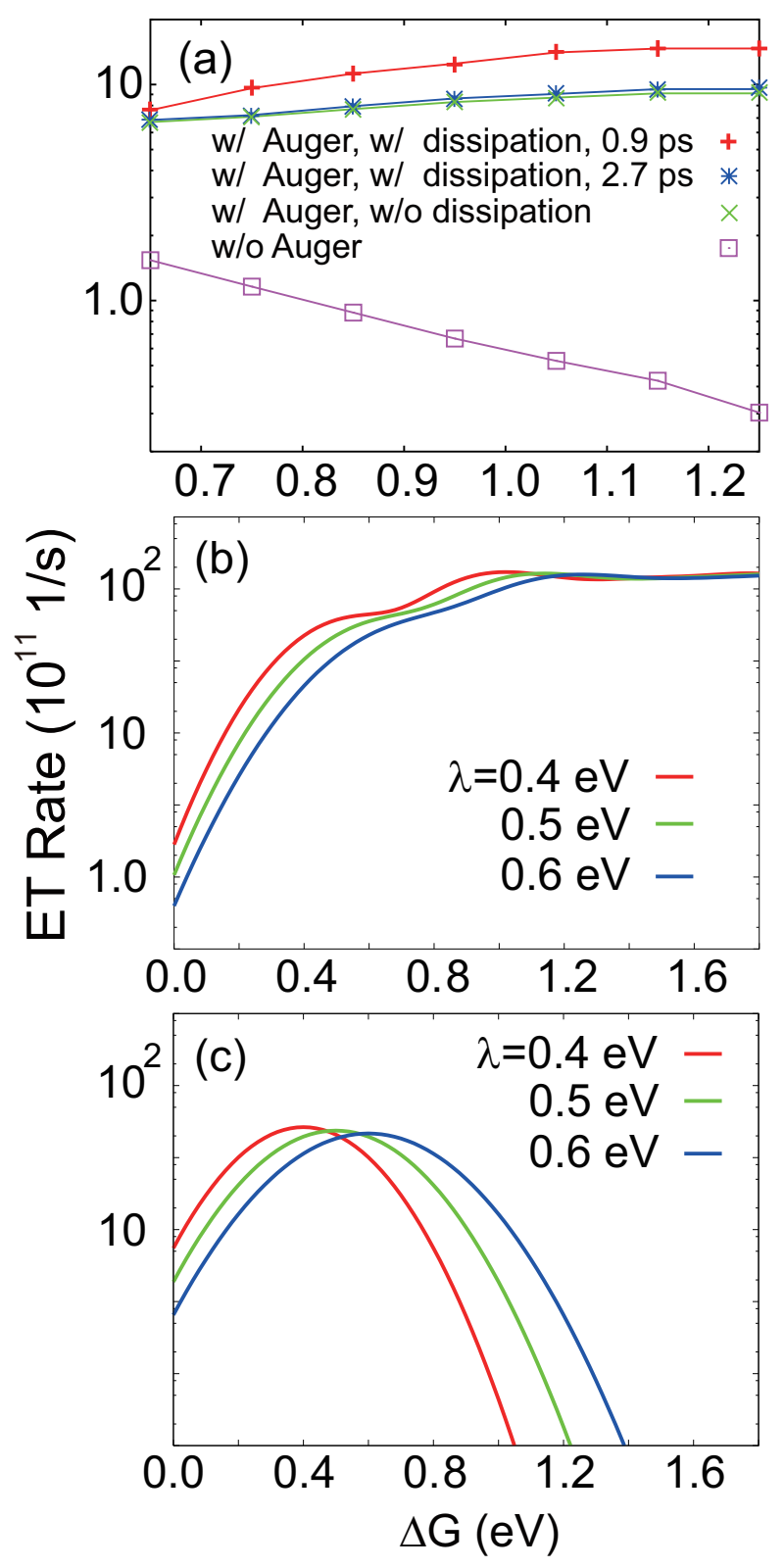

Figure 5: (a) ET rates as a function of the driving force $\Delta G$. The panel compares four cases with and without the Auger interaction, influenced by phonon dissipation on different timescales. The AAET rates increase with $\Delta G$ and reach a plateau, while the conventional ET rate monotonically decreases with increasing $\Delta G$, corresponding to the Marcus inverted region. Phonon dissipation slightly increases the ET rate, since it quenches the hole energy and allows repeated hole excitation. (b) ET rates calculated from the extended Marcus theory. The AAET rates have no inverted region and reproduce the experimental plateau. (c) ET rates calculated with the single acceptor state, i.e. $\rho(E)=\delta(E)$ in eq.(4). The resulting rates decrease at large $\Delta G$, clearly showing the Marcus inverted region.

The ET rate is obtained as the inverse of the ET time estimated as in Fig.S2. , $k_{\mathrm{ET}}=$ 
$1 / \tau_{\mathrm{ET}}$. Figure $5(\mathrm{a})$ shows the ET rates for a wide range of $\Delta G$ values. Four distinct situations are compared: (1) AAET accompanied by charge-phonon energy dissipation of the realistic timescale, $\tau_{\text {diss }}=0.9$ ps. (2) AAET accompanied by charge-phonon energy dissipation of a longer timescale, $\tau_{\text {diss }}=2.7$ ps. (3) AAET without charge-phonon energy dissipation. (4) Conventional ET without the hole dynamics. The last case includes only the QD LUMO and MB LUMO electron states, and the electronic energy is deposited into phonons only.

The AAET rates, cases (1)-(3), vary little with $\Delta G$ and reach plateaus around $\Delta G=1.05$ $\mathrm{eV}$. Because the hole excitation accompanies the ET, the QD LUMO to MB LUMO gap is offset by the hole excitation, and the true D-A gap changes little with increasing $\Delta G$. The ET rate keeps growing because the density of final states, determined by the VB DOS, increases. The plateau is then reached because the VB DOS saturates as in Fig. 2(a). Taking into account the experimental fact that the ET rate increases with decreasing QD size, for most QD materials and molecular acceptors, the current ET rates compare well with the experimental ET rates. ${ }^{14}$ If the ET had followed traditional Marcus theory, the rates would have changed by two orders for the shown $\Delta G$ range. Case (4) supports this fact. Our time-domain $a b$ initio simulations confirm that the Auger excitation of the hole eliminates the inverted region.

Phonon dissipation plays a minor role in determining the AAET rate. The ET rates with and without dissipation, cases (1) and (3), differ slightly, and the ET rates with the dissipation time $\tau_{\text {diss }}=2.7$ ps already converge to the ET rates without any dissipation. Energy dissipation by phonons accelerates the ET because the dissipation of the excited hole energy allows additional hole excitation. This fact is supported by the growing difference in the ET rates of cases (1) and (3) with increasing $\Delta G$. Larger $\Delta G$ requires more effective Auger hole excitation, and the dissipation becomes more important in allowing additional hole excitation during the ET.

In addition to the time-domain ab initio simulation, we derive the extended Marcus ET rate theory involving the Auger hole excitation based on Fermi's golden rule expression 
starting from the model Hamiltonian as explained in the Supporting Information. Assuming that the average NA coupling constant $\left|H_{r p}\right|$ is approximately independent of energy, we get the final form of the rate

$$
k_{E T}=\frac{2 \pi}{\hbar} \frac{\left|H_{r p}\right|^{2}}{\sqrt{4 \pi \lambda k T}} \int d E \rho(E) \exp \left(-\frac{(\lambda+E-\Delta G)^{2}}{4 \lambda k T}\right),
$$

with the temperature $T=300 \mathrm{~K} . k$ and $\lambda$ are the Boltzmann factor and a reorganization energy of phonons, respectively. The density of states $\rho(E)$ is the density of the final acceptor states as a function of the hole excitation energy $E$. Thus, $-E+\Delta G$ corresponds to the true driving force. Note that the current simulation and analytic model are formulated in the adiabatic representation, in which all Coulomb interactions are diagonalized out to obtain the adiabatic state, that is, eigen states of the electronic Hamiltonian. Therefore, the coupling $H_{r p}$ is the non-adiabatic coupling rather than the Coulomb coupling. We emphasize that our final expression (4) includes neither adjustable parameters nor scaling factors, and is specified completely by the clear physical quantities obtained from the timedependent $a b$ initio calculations. We numerically perform the integration in Eq. (4) using the averaged values of the DOS $\rho(E)$ and the NA coupling constant $\left|H_{r p}(E)\right|^{2}$ obtained for the current $\mathrm{QD} / \mathrm{MB}$ complex at the ambient temperature by our time-dependent ab initio MD simulations, $\left|H_{r p}(E)\right|=1.00 \times 10^{-2} \mathrm{eV}$. The calculated AAET rates are shown in Fig. 5(b). The rates exhibit no inverted region and saturate at large $\Delta G$, reproducing the plateau observed in both the experiment and direct time-domain ab initio calculations. The analytic model indicates that dense VB states and strong Coulomb electron-hole interaction in a donor nanomaterial can induce an efficient AAET. As shown in Fig.5(c), the ET rates calculated with the single acceptor state, i.e. $\rho(E)=\delta(E)$ are qualitatively different; they decrease at large $\Delta G$, clearly showing the Marcus inverted region.

We conclude that Auger-type hole excitation in the electron donor material eliminates the Marcus inverted region. We have examined the AAET mechanism by the direct time-domain 
ab initio simulation performed on the CdSe QD/MB complex. ${ }^{14}$ We have demonstrated that the ET is accompanied by promotion of the hole from the QD HOMO deeper into the QD VB. The simulated ET dynamics exhibits two time-scales. The initial Gaussian dynamics is associated with the direct electron-hole energy exchange, while the slower exponential component corresponds to the charge-phonon energy relaxation.

We have also derived an extended Marcus rate theory for the ET to include the Auger hole excitation, and have reproduced analytically the experimental plateau of the AAET rate at large driving forces. The analytic results are based solely on ab initio input, without any adjustable parameters.

We have found that phonons play competitive and important roles. A notable fraction of the electron energy flows directly to phonons, bypassing the hole, thereby enabling the traditional phonon-assisted ET mechanism. In addition, phonons dissipate the excited hole energy, allowing the hole to accommodate additional electron energy. Having lost some energy to phonons, the hole can be excited again, facilitating AAET.

In the particular system under investigation, the QD electron donor state is localized opposite to the adsorbed molecular acceptor. By forcing the electron to travel across the QD, this situation further strengthens the electron-hole interaction, which is already enhanced by the quantum confinement.

It is possible that acceptor molecules are separated from QD donors by ligands. Ligands would decrease the donor-acceptor electronic coupling and create an energy barrier, slowing down the ET. The electron transferred to the acceptor molecule will interact with the hole much less; the electron-hole interaction will be strong only at the initial stage of the ET, making the Auger process less efficient. In addition, removing the hole from the QD core by ligand trapping should eliminate the AAET mechanism altogether and restore the Marcus inverted regime. The Marcus inverted region should be restored as the QD size approaches and exceeds the exciton Bohr radius of the corresponding bulk semiconductor. The VB DOS would be too low to support AAET in very small nanoclusters, whose properties are 
similar to properties of molecules. The qualitative aspects of AAET should not change in the presence of multiple acceptor molecules adsorbed on the QD surface. Assuming that these molecules do not form strongly interacting dimers and larger clusters, they should just provide parallel channels for AAET.

Auger-type phenomena are very common in nanoscale systems due to strong Coulomb interactions induced by quantum confinement and due to high densities of states. Molecules also exhibit strong electron-hole interactions, but have low state densities. Bulk materials have high state densities, but exhibit weak Coulomb interactions due to dielectric screening. The reported insights into the AAET process stimulate ideas on how this recently discovered phenomenon can be utilized for controlling charge transfer and relaxation processes in nanoscale materials.

\section{Acknowledgment}

KHD acknowledges financial support from JST (PRESTO), and Grant-in-Aids for Scientific Research from Japan Society for the Promotion of Science (KAKENHI), Grant No. 24750016. OVP acknowledges funding from from the Office of Basic Energy Sciences of the U.S. Department of Energy, Grant DE-SC0006527.

Supporting Information Available: Complete theoretical derivations, simulation details, and additional figures. This information is available free of charge via the Internet at http://pubs.acs.org.

\section{References}

(1) Kobayashi, Y.; Nonoguchi, Y.; Wang, L.; Kawai, T.; Tamai, N. Dual Transient Bleaching of $\mathrm{Au} / \mathrm{PbS}$ Hybrid Core/Shell Nanoparticles J. Phys. Chem. Lett. 2012, 3, 11111116. 
(2) Kobayashi, Y.; Nishimura, T.; Yamaguchi, H.; Tamai, N. Effect of Surface Defects on Auger Recombination in Colloidal CdS Quantum Dots J. Phys. Chem. Lett. 2011, 2, $1051-1055$.

(3) Chen, J.; Schmitz, A.; Inerbaev, T.; Meng, Q.; Kilina, S.; Tretiak, S.; Kilin, D. S. First-Principles Study of pn-Doped Silicon Quantum Dots: Charge Transfer, Energy Dissipation, and Time-Resolved Emission J. Phys. Chem. Lett. 2013, 4, 2906-2913.

(4) Long, R.; English, N. J.; Prezhdo, O. V. Minimizing ElectronHole Recombination on TiO2 Sensitized with PbSe Quantum Dots: Time-Domain Ab Initio Analysis J. Phys. Chem. Lett. 2014, 5, 2941-2946.

(5) Rego, L. G. C.; Batista, V. S. Quantum Dynamics Simulations of Interfacial Electron Transfer in Sensitized TiO2 Semiconductors J. Am. Chem. Soc. 2005, 125, 7989-7997.

(6) Abuabara, S. G.; Rego, L. G. C.; Batista, V. S. Influence of Thermal Fluctuations on Interfacial Electron Transfer in Functionalized TiO2 Semiconductors J. Am. Chem. Soc. 2005, 127, 18234-18242.

(7) Long, R.; Prezhdo, O. V. Instantaneous Generation of Charge-Separated State on TiO2 Surface Sensitized with Plasmonic Nanoparticles J. Am. Chem. Soc. 2014, 136, $4343-4354$

(8) Nguyen, P. D.; Ding, F.; Fischer, S. A.; Liang, W.; Li, X. Solvated First-Principles Excited-State Charge-Transfer Dynamics with Time-Dependent Polarizable Continuum Model and Solvent Dielectric Relaxation J. Phys. Chem. Lett. 2012, 3, 28982904.

(9) Barbara, P. F.; Meyer, T. J.; Ratner, M. A. Contemporary Issues in Electron Transfer Research J. Phys. Chem. 1996, 100, 13148-13168. 
(10) Marcus, R.; Sutin, N. Electron Transfers in Chemistry and Biology Biochim. Biophys. Acta 1985, 811, 265-322.

(11) Marcus, R. A. On the Theory of Oxidation-Reduction Reactions Involving Electron Transfer J. Chem. Phys. 1956, 24, 966-978.

(12) Closs, G. L.; Miller, J. R. Intramolecular Long-Distance Electron-Transfer in OrganicMolecules Science 1988, 240, 440-447.

(13) Hendry, E.; Koeberg, M.; Wang, F.; Zhang, H.; Donega, C. M.; Vanmaekelbergh, D.; Bonn, M. Direct Observation of Electron-to-Hole Energy Transfer in CdSe Quantum Dots Phys. Rev. Lett. 2006, 96, 057408.

(14) Zhu, H.; Yang, Y.; Hyeon-Deuk, K.; Califano, M.; Song, N.; Wang, Y.; Zhang, W.; Prezhdo, O. V.; Lian, T. Auger-Assisted Electron Transfer from Photoexcited Semiconductor Quantum Dots Nano Lett. 2014, 14, 1263-1269.

(15) Fischer, S. A.; Lingerfelt, D. B.; May, J. W.; Li, X. Non-adiabatic molecular dynamics investigation of photoionization state formation and lifetime in $\mathrm{Mn} 2+$-doped $\mathrm{ZnO}$ quantum dots Phys. Chem. Chem. Phys. 2014, 16, 17507-17514.

(16) Muljarov, E. A.; Takagahara, T.; Zimmermann, R. Phonon-induced exciton dephasing in quantum dot molecules Phys. Rev. Lett. 2005, 95, 177405.

(17) Hyeon-Deuk, K.; Prezhdo, O. V. Multiple Exciton Generation and Recombination Dynamics in Small Si and CdSe Quantum Dots: an Ab Initio Time-Domain Study ACS Nano 2012, 6, 1239-1250.

(18) Hyeon-Deuk, K.; Prezhdo, O. V. Photoexcited electron and hole dynamics in semiconductor quantum dots: phonon-induced relaxation, dephasing, multiple exciton generation and recombination J. Phys. Cond. Matt. 2012, 24, 363201. 
(19) Kilina, S. V.; Kilin, D. S.; Prezhdo, O. V. Breaking the Phonon Bottleneck in PbSe and CdSe Quantum Dots: Time-Domain Density Functional Theory of Charge Carrier Relaxation ACS Nano 2009, 3, 93-99.

(20) Jaeger, H. M.; Hyeon-Deuk, K.; Prezhdo, O. V. Exciton Multiplication from First Principles Acc. Chem. Res. 2013, 46, 1280-1289.

(21) Hyeon-Deuk, K.; Prezhdo, O. V. Time-domain ab initio study of Auger and phononassisted Auger processes in a semiconductor quantum dot Nano Lett. 2011, 11, 18451850.

(22) Hyeon-Deuk, K.; Kobayashi, Y.; Tamai, N. Evidence of Phonon-Assisted Auger Recombination and Multiple Exciton Generation in Semiconductor Quantum Dots Revealed by Temperature-Dependent Phonon Dynamics J. Phys. Chem. Lett. 2014, 5, 99-105.

(23) Kilina, S. V.; Neukirch, A. J.; Habenicht, B. F.; Kilin, D. S.; Prezhdo, O. V. Quantum Zeno Effect Rationalizes the Phonon Bottleneck in Semiconductor Quantum Dots Phys. Rev. Lett. 2013, 110, 180404.

(24) Maniscalco, S.; Piilo, J.; Suominen, K.-A. Zeno and Anti-Zeno Effects for Quantum Brownian Motion Phys. Rev. Lett. 2006, 97, 130402.

(25) Streed, E. W.; Mun, J.; Boyd, M.; Campbell, G. K.; Medley, P.; Ketterle, W.; Pritchard, D. E. Continuous and Pulsed Quantum Zeno Effect Phys. Rev. Lett. 2006, 97, 260402. 


\title{
Supporting Information for "Ab initio Analysis of
}

\section{Auger-Assisted Electron Transfer"}

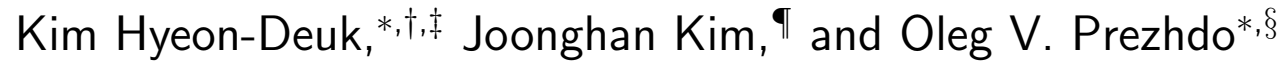 \\ Department of Chemistry, Kyoto University, Kyoto, 606-8502, Japan, Japan Science and \\ Technology Agency, PRESTO, 4-1-8 Honcho, Kawaguchi, Saitama, 332-0012, Japan, \\ Department of Chemistry, The Catholic University of Korea, Bucheon, Gyunggi-do \\ 420-743, Korea, and Department of Chemistry, University of Southern California, Los
}

Angeles, CA 90089, USA

E-mail: kim@kuchem.kyoto-u.ac.jp; prezhdo@usc.edu

\footnotetext{
${ }^{*}$ To whom correspondence should be addressed

${ }^{\dagger}$ Department of Chemistry, Kyoto University, Kyoto, 606-8502, Japan

$\ddagger$ Japan Science and Technology Agency, PRESTO, 4-1-8 Honcho, Kawaguchi, Saitama, 332-0012, Japan

IDepartment of Chemistry, The Catholic University of Korea, Bucheon, Gyunggi-do 420-743, Korea

$\S$ Department of Chemistry, University of Southern California, Los Angeles, CA 90089, USA
} 


\section{Non-adiabatic molecular dynamics with time-dependent density functional theory}

The electronic structure and adiabatic molecular dynamics(MD) are computed with VASP. ${ }^{1}$ The PW91 density functional and projector-augmented-wave pseudopotentials are used. The electron-hole interaction in the quantum $\operatorname{dot}(\mathrm{QD}) /$ methylene blue(MB) complex is included implicitly by the density functional theory(DFT) exchange-correlation functional. The QD/MB complex is fully optimized at zero temperature and heated up to an ambient temperature by repeated velocity rescaling. A 4 ps microcanonical trajectory is calculated on the ground electronic state by the ab initio MD using the Verlet algorithm with a 1 fs time-step. The initial conditions for the non-adiabatic MD(NAMD) are sampled from this trajectory.

In order to account for the Auger hole excitation accompanying the electron transfer(ET), we adopt the exciton representation. Figure S1 shows the exciton basis states included in the calculation. The QD band gap excitation is the donor state: Initially, the electron is in the LUMO, while the hole is in the HOMO. All the other states are acceptor states, with the electron transferred to the MB LUMO (indicated by the red line). The multiple acceptor states correspond to different energies of the Auger hole excitation. The number of the final acceptor states, $N_{\mathrm{VB}}=43$, equals to the number of the QD VB states considered here. $\Delta G$ is the driving force of the ET. It is defined as the energy difference between the donor and acceptor states represented by the QD LUMO and the MB LUMO, respectively. The $\Delta G$ dependence of the Auger-assisted electron transfer (AAET) process is investigated by scaling the original QD LUMO-MB LUMO energy difference. The shown VB states are above the MB HOMO, such that the hole is never transferred to the MB and remains in the QD, as in the experiment. ${ }^{2}$

The NA dynamics simulations for the AAET are performed by combining the timedomain DFT(TDDFT) with the NAMD. The TDDFT calculations are performed in the 
Kohn-Sham (KS) representation. The adiabatic KS orbitals depend on phonon coordinates, and transitions between different adiabatic states occur due to the NA couplings, as will be explained below. The time-dependent diabatic single-electron $\mathrm{KS}$ orbitals $\phi_{p}(\mathbf{r}, t)$ are evolved using the time-dependent KS equations

$$
i \hbar \frac{\partial \phi_{p}(\mathbf{r}, t)}{\partial t}=H(\phi(\mathbf{r}, t)) \phi_{p}(\mathbf{r}, t), \quad p=1, \ldots, N_{e}
$$

where $N_{e}$ is the number of electrons. The equations for all $p$ are coupled through the dependence of the Hamiltonian $H$ on the electron density obtained by summing over all the KS orbitals occupied by the $N_{e}$ electrons. Expanding the time-dependent diabatic KS orbitals $\phi_{p}(\mathbf{r}, t)$ by the adiabatic KS orbital basis $\tilde{\phi}_{k}(\mathbf{r} ; \mathbf{R})$,

$$
\phi_{p}(\mathbf{r}, t)=\sum_{k=1}^{N_{e}} c_{p k}(t)\left|\tilde{\phi}_{k}(\mathbf{r} ; \mathbf{R})\right\rangle,
$$

leads the time-dependent KS eq.(1) to the equations of motion(EOMs) for the expansion coefficients ${ }^{3}$

$$
i \hbar \frac{\partial c_{p k}(t)}{\partial t}=\sum_{m=1}^{N_{e}} c_{p m}(t)\left(\epsilon_{m} \delta_{k m}-i \hbar \mathbf{d}_{k m} \cdot \dot{\mathbf{R}}\right) .
$$

The adiabatic KS orbitals $\tilde{\phi}_{k}(\mathbf{r} ; \mathbf{R})$ are obtained with the DFT for atomic positions at each moment along the $a b$ initio MD trajectory. The NA coupling expresses the electron-phonon interaction

$$
\mathbf{d}_{k m} \cdot \dot{\mathbf{R}}=\left\langle\tilde{\phi}_{k}(\mathbf{r} ; \mathbf{R})\left|\nabla_{\mathbf{R}}\right| \tilde{\phi}_{m}(\mathbf{r} ; \mathbf{R})\right\rangle \cdot \dot{\mathbf{R}}=\left\langle\tilde{\phi}_{k}(\mathbf{r} ; \mathbf{R})\left|\frac{\partial}{\partial t}\right| \tilde{\phi}_{m}(\mathbf{r} ; \mathbf{R})\right\rangle
$$

It stems from the dependence of the adiabatic KS orbitals on the phonon dynamics $\mathbf{R}(t)$. Since the NA coupling is proportional to the nuclear velocity $\dot{\mathbf{R}}$, NA transitions would never happen under the Born-Oppenheimer approximation. 
We represent the above TDDFT equations in the second quantization notation to include the ground and single exciton (SE) states, $\left|\Phi_{g}(\mathbf{r} ; \mathbf{R})\right\rangle$ and $\left|\Phi_{S E}^{i, j}(\mathbf{r} ; \mathbf{R})\right\rangle$, respectively. ${ }^{4-6}$ The SEs are obtained by applying the raising and lowering operators to the ground state,

$$
\left|\Phi_{S E}^{i, j}\right\rangle=\hat{a}_{i}^{\dagger} \hat{a}_{j}\left|\Phi_{g}\right\rangle
$$

The electron creation and annihilation operators, $\hat{a}_{i}^{\dagger}$ and $\hat{a}_{j}$, generate and annihilate an electron in the $i$ th and $j$ th adiabatic KS orbitals, respectively. The total wave function is then expanded as

$$
|\Psi(t)\rangle=C_{g}(t)\left|\Phi_{g}\right\rangle+\sum_{i, j} C_{S E}^{i, j}(t)\left|\Phi_{S E}^{i, j}\right\rangle
$$

Substitution of eq.(6) into the time-dependent Schrödinger equation leads to the EOMs for the expansion coefficients appearing in eq.(6),

$$
i \hbar \frac{\partial C_{X}(t)}{\partial t}=C_{X}(t) E_{X}-i \hbar C_{g}(t) \mathbf{d}_{X ; g} \cdot \dot{\mathbf{R}}-i \hbar \sum_{i^{\prime}, j^{\prime}} C_{S E}^{i^{\prime}, j^{\prime}}(t) \mathbf{d}_{X ; S E, i^{\prime}, j^{\prime}} \cdot \dot{\mathbf{R}}
$$

where $X$ now corresponds to either the ground or SE state, and $E_{X}$ is the state energy. The NA couplings introduced by

$$
\mathbf{d}_{X ; Y} \cdot \dot{\mathbf{R}} \equiv\left\langle\Phi_{X}\left|\nabla_{\mathbf{R}}\right| \Phi_{Y}\right\rangle \cdot \dot{\mathbf{R}}=\left\langle\Phi_{X}\left|\frac{\partial}{\partial t}\right| \Phi_{Y}\right\rangle
$$

induce NA transitions between the states in the exciton basis. The NA couplings, eqs.(4) and (8), as well as the EOMs (3) and (7), are one-to-one correspondence. ${ }^{2,4-7}$

The atomistic simulation of the ET dynamics is performed by directly solving eq.(7) with the time-dependent NA couplings and energies obtained by the ab initio MD simulation. The initial SE state is created with the electron in the QD LUMO and the hole in the QD HOMO. Since only one particle, the electron or the hole, can make a transition by a single operation 
of the NA coupling (8), the AAET is completed by a two-step transition caused by double operations of the NA coupling. The intermediate state obtained after the first operation of the NA coupling can be interpreted as a virtual state, for instance, similar to Raman spectroscopy.

We further introduced energy dissipation to phonon modes by the exponential decay function, $\exp \left(-t / \tau_{\text {diss }}\right)$, where $t$ is the simulation time and $\tau_{\text {diss }}$ is set equal to 0.9 ps unless explicitly specified otherwise. The characteristic relaxation time, $0.9 \mathrm{ps}$, corresponds to the typical relaxation time of an excited hole in the isolated CdSe QD. ${ }^{3}$ In each NA transition step, we multiplied population of each state other than the Acceptor 1 state drawn in Fig.S1 by this simple exponential decay function. The decreased population in the each state flows into the Acceptor 1 state to preserve the total population. The conventional ET including only the Donor and Acceptor 1 states in Fig.S1 are calculated with the the phonon dephasing effect for comparison with the AAET. ${ }^{8}$

\section{Analytic theory of AAET}

In addition to the time-domain $a b$ initio simulation, we derive the extended Marcus rate theory for the ET involving the Auger hole excitation starting from the model Hamiltonian. The exciton Hamiltonian is introduced as

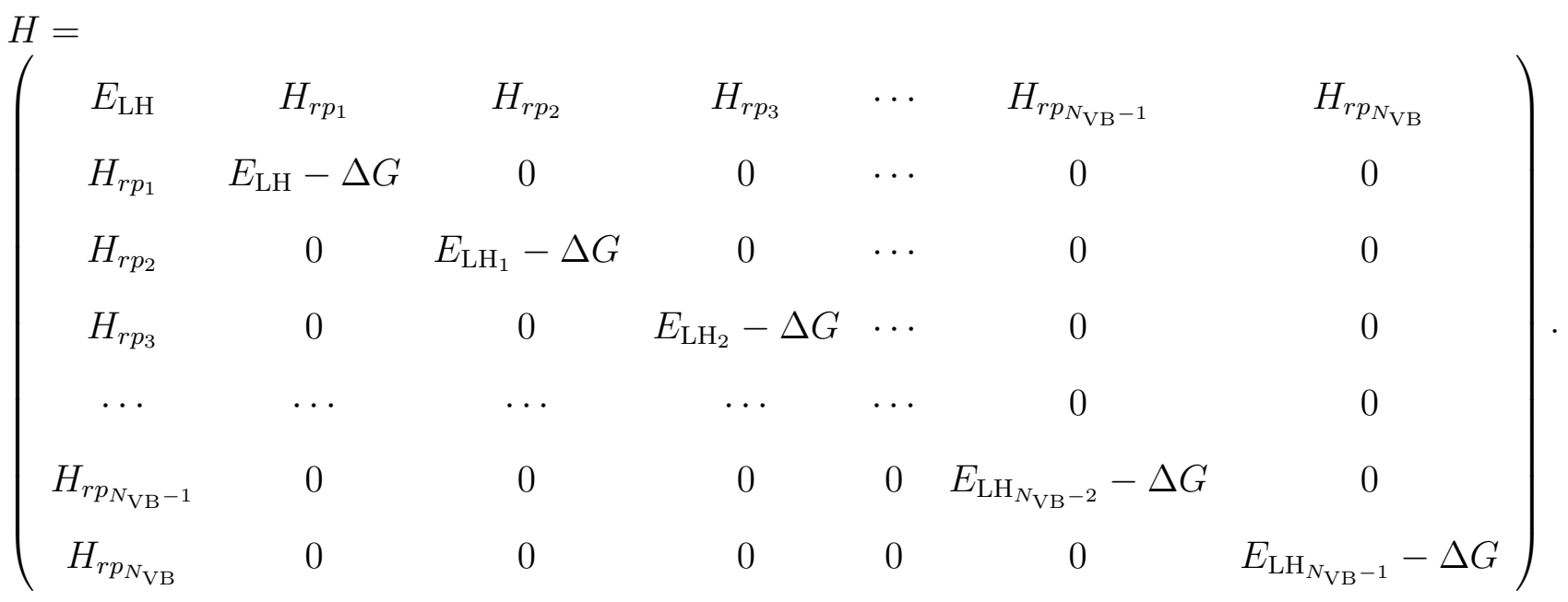


Here, $E_{\mathrm{LH}}$ is the QD LUMO-HOMO band gap energy, and $E_{\mathrm{LH}_{i}}$ indicates an energy between the QD LUMO and HOMO-ith VB states. These exciton states are all calculated based on the adiabatic KS orbitals, so the off-diagonal components of the Hamiltonian should be the NA couplings connecting the adiabatic exciton states rather than Coulomb couplings connecting diabatic states in a conventional description of the Auger-type process. We emphasize that the $a b$ initio description necessitates the adiabatic representation, in which all Coulomb interaction terms are diagonalized out. Since we focus only on the ET from the QD to the adsorbed MB, all NA transitions among the acceptor states are neglected in the analytic Marcus theory. The NA coupling terms are thus

$$
H_{r p_{i}} \equiv-i \hbar \mathbf{d}_{r p_{i}} \cdot \dot{\mathbf{R}}
$$

The right-hand side arises from the dependence of the adiabatic KS orbitals $\tilde{\phi}_{k}(\mathbf{r} ; \mathbf{R})$ on the phonon dynamics $\mathbf{R}(t)$ :

$$
\mathbf{d}_{r p_{i}} \cdot \dot{\mathbf{R}}=\left\langle\tilde{\phi}_{r}(\mathbf{r} ; \mathbf{R})\left|\nabla_{\mathbf{R}}\right| \tilde{\phi}_{p_{i}}(\mathbf{r} ; \mathbf{R})\right\rangle \cdot \dot{\mathbf{R}}=\left\langle\tilde{\phi}_{r}(\mathbf{r} ; \mathbf{R})\left|\frac{\partial}{\partial t}\right| \tilde{\phi}_{p_{i}}(\mathbf{r} ; \mathbf{R})\right\rangle .
$$

Here, $\tilde{\phi}_{r}(\mathbf{r} ; \mathbf{R})$ expresses the adiabatic KS orbital of the donor state (the band gap state in Fig.S1), while $\tilde{\phi}_{p_{i}}(\mathbf{r} ; \mathbf{R})$ corresponds to the adiabatic KS orbital of the $i$ th acceptor state.

The perturbative treatment of eq.(3) with $N_{e}=N_{\mathrm{VB}}+1$ and with $\epsilon_{m}$ corresponding to the $m$ th diagonal component of the Hamiltonian (9) results in Fermi's golden rule expression for the ET rate just as in Refs. ${ }^{9}$ and, ${ }^{10}$

$$
k_{E T}=\frac{2 \pi}{\hbar} \sum_{i=1}^{N_{\mathrm{VB}}}\left|H_{r p_{i}}\right|^{2} \mathrm{FC}_{i}
$$

The summation is carried out over all possible acceptor states. $\mathrm{FC}_{i}$ is the Franck-Condon 
factor which incorporates the energy conservation $\delta\left(E_{\mathrm{LH}}-E_{\mathrm{LH}_{i-1}}+\Delta G\right)$ and the overlap integrals of the vibrational wave functions for the donor and $i$ th acceptor states. When all phonon modes have relatively small frequencies and are treated classically using a free energy of reorganization, the above Fermi's golden rule expression leads to the Marcus-type rate expression just as in Refs. ${ }^{11}$ and; ${ }^{12}$

$$
k_{E T}=\frac{2 \pi}{\hbar} \frac{1}{\sqrt{4 \pi \lambda k T}} \int d E\left|H_{r p}(E)\right|^{2} \rho(E) \exp \left(-\frac{(\lambda+E-\Delta G)^{2}}{4 \lambda k T}\right) .
$$

In the AAET case, $E$ is introduced as an energy of the hole excitation, $E \equiv E_{\mathrm{LH}_{i}}-E_{\mathrm{LH}}$. Thus, $-E+\Delta G$ corresponds to a true driving force. $k, T$ and $\lambda$ are the Boltzmann factor, temperature, and a reorganization energy of phonons, respectively. $\left|H_{r p}(E)\right|^{2}$ is the averaged NA coupling constant between the energies $E$ and $E+\Delta E$ for a given $\Delta G$;

$$
\left|H_{r p}(E)\right|^{2} \equiv \frac{1}{N_{\text {states }}} \sum_{i \in[E, E+\Delta E]}\left|H_{r p_{i}}\right|^{2},
$$

where $N_{\text {states }}$ is the number of states between the energies $E$ and $E+\Delta E$. The density of states $\rho(E)$ stems from the energy conservation term $\delta\left(E_{\mathrm{LH}}-E_{\mathrm{LH}_{i-1}}+\Delta G\right)$ in the $\mathrm{FC}_{i}$ factor of eq.(12). It is the density of the final acceptor states as a function of the hole excitation energy $E$. If we did not take into account the Auger hole excitation, the final acceptor state would be restricted to the Acceptor 1 state in Fig.S1, and $\rho(E)$ should be replaced by the simple delta function, $\delta(E)$.

We compare the two ET cases with and without the Auger hole excitation, and check whether the Marcus inverted region appears or not. In order to calculate the ET rate (13), we assume that the average NA coupling constant $\left|H_{r p}(E)\right|^{2}$ is approximately independent of energy, leading to the final form of the rate

$$
k_{E T}=\frac{2 \pi}{\hbar} \frac{\left|H_{r p}\right|^{2}}{\sqrt{4 \pi \lambda k T}} \int d E \rho(E) \exp \left(-\frac{(\lambda+E-\Delta G)^{2}}{4 \lambda k T}\right)
$$


Temperature $T$ is set as $300 \mathrm{~K} .\left|H_{r p}\right|^{2}$ is now independent of both $E$ and $\Delta G$, which is partially related to the $\Delta G$ scaling explained in Fig.S1. We emphasize that our final expression (15) includes neither adjustable parameters nor scaling factors, and is specified completely by the clear physical quantities obtained from the time-dependent $a b$ initio calculations. 


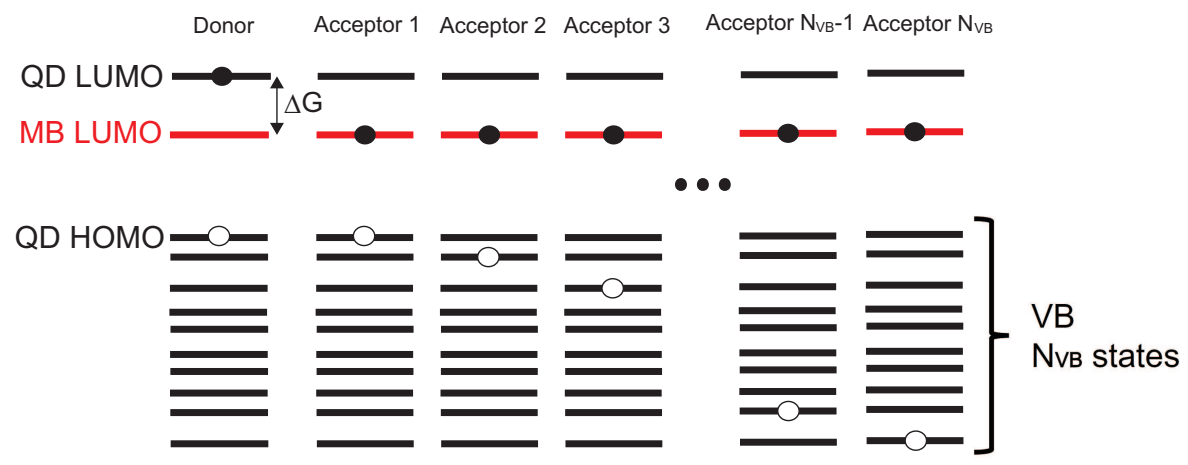

Figure S1: The exciton basis used in the current simulations. The donor state is the QD band gap excitation. The electron is transferred to the MB LUMO (red line). A large number of acceptor states are included, corresponding to different hole excitations within the QD VB. The ET driving force, $\Delta G$, is defined as the energy difference between the QD LUMO and the MB LUMO. 


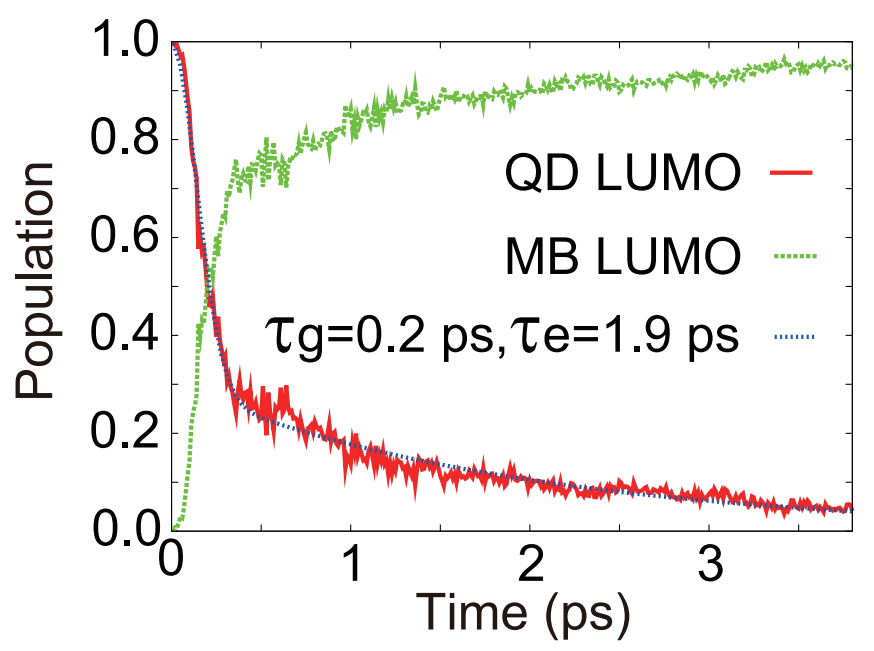

Figure S2 : Representative dynamics of the QD LUMO and MB LUMO populations reflecting the ET. The time-dependent populations can be fitted by the combined function of the Gaussian and exponential, $A \exp \left(-t^{2} / \tau_{g}^{2}\right)+(1-A) \exp \left(-t / \tau_{e}\right)$. The dynamics exhibits two distinct timescales, $\tau_{g}$ and $\tau_{e}$. The initial Gaussian dynamics is associated with the direct electron-hole energy exchange, while the slower exponential component corresponds to the charge-phonon energy relaxation. We estimated the ET time, $\tau_{\mathrm{ET}}$, as the weighted average of the two timescales, $t_{\mathrm{ET}}=A \tau_{g}+(1-A) \tau_{e}$. For example, the ET time for the shown process is calculated as $0.71 \mathrm{ps}$, with $A=0.7$, and the Gaussian and exponential time constants given in the figure. 


\section{References}

(1) Kresse, G.; Furthmuller, J. Efficiency of ab-initio total energy calculations for metals and semiconductors using a plane-wave basis set Comp. Mat. Sci. 1996, 6, 15-50.

(2) Zhu, H.; Yang, Y.; Hyeon-Deuk, K.; Califano, M.; Song, N.; Wang, Y.; Zhang, W.; Prezhdo, O. V.; Lian, T. Auger-Assisted Electron Transfer from Photoexcited Semiconductor Quantum Dots Nano Lett. 2014, 14, 1263-1269.

(3) Kilina, S. V.; Neukirch, A. J.; Habenicht, B. F.; Kilin, O. V., D. S. Prezhdo Quantum Zeno Effect Rationalizes the Phonon Bottleneck in Semiconductor Quantum Dots Phys. Rev. Lett. 2013, 110, 180404.

(4) Hyeon-Deuk, K.; Prezhdo, O. V. Time-domain ab initio study of Auger and phononassisted Auger processes in a semiconductor quantum dot Nano Lett. 2011, 11, 18451850.

(5) Hyeon-Deuk, K.; Prezhdo, O. V. Multiple Exciton Generation and Recombination Dynamics in Small Si and CdSe Quantum Dots: an Ab Initio Time-Domain Study ACS Nano 2012, 6, 1239-1250.

(6) Hyeon-Deuk, K.; Kobayashi, Y.; Tamai, N. Evidence of Phonon-Assisted Auger Recombination and Multiple Exciton Generation in Semiconductor Quantum Dots Revealed by Temperature-Dependent Phonon Dynamics J. Phys. Chem. Lett. 2014, 5, 99-105.

(7) Craig, C. F.; Duncan, W. R.; Prezhdo, O. V. Trajectory Surface Hopping in the Time-Dependent Kohn-Sham Approach for Electron-Nuclear Dynamics Phys. Rev. Lett. 2005, 95, 163001.

(8) Habenicht, B. F.; Karnisaka, H.; Yarnashita, K.; Prezhdo, O. V. Ab initio study of 
vibrational dephasing of electronic excitations in semiconducting carbon nanotubes Nano Lett. 2007, 7, 3260-3265.

(9) Mikkelsen, K. V.; Ratner, M. A. Electron Tunneling in Solid-State Electron-Transfer Reactions Chem. Rev. 1987, 87, 113-153.

(10) Kestner, N. R.; Logan, J.; Jortner, J. Thermal Electron Transfer Reactions in Polar Solvents J. Phys. Chem. 1974, 74, 2148-2166.

(11) Marcus, R.; Sutin, N. Electron Transfers in Chemistry and Biology Biochim. Biophys. Acta $\mathbf{1 9 8 5}, 811,265-322$.

(12) Gao, Y. Q.; Georgievskii, Y.; Marcus, R. A. On the theory of electron transfer reactions at semiconductor electrode/liquid interfaces J. Chem. Phys. 2000, 112, 3358-3369. 